\title{
Food Product Traceability by Using Automated Identification Technologies
}

\author{
Ivana Šenk, Gordana Ostojić, Laslo Tarjan, \\ Stevan Stankovski, and Milovan Lazarević \\ University of Novi Sad, Faculty of Technical Sciences, Trg Dositeja Obradovića 6, 21000 \\ Novi Sad, Serbia \\ \{ivanas, goca, laci, stevan, laza\} @uns.ac.rs
}

\begin{abstract}
Food product traceability from harvesting, through food processing to the final food product and through the retailer to the end consumer is a significant process that has to ensure food quality and safety. The traceability enables the end consumer to get information from all previous stages of the food product, leading back to the food origin. In this way, the consumer can get more information on the specific product, and thus make a decision on buying the product that suits his needs best. In each stage of the food product transformation, important data are generated for the subsequent chain participants. Every participant should have access to certain data of interest to them. This can be achieved by using automated identification technologies, like RFID (Radio Frequency IDentification) and two-dimensional barcode, which allow faster data acquisition, recording and reading processes than the traditional means, and provide up-to-date information in each product stage. Furthermore, these technologies allow the possibility to record large amounts of data for each specific product, and interconnect all the data in a database. This paper discusses the process of providing traceability of food products, recording, transmitting and reading of significant data in specific stages of food product chain, with the application of automated identification technologies, including the possibility of obtaining additional data from a database, according to appropriate access level of each participant in the chain. Advantages and disadvantages of automated identification technologies are discussed, with the proposition for using specific technologies in certain food product stages.
\end{abstract}

Keywords: Food traceability, RFID, 2D barcode, QR code.

\section{Introduction}

Great competition within the food market forces the food producers to introduce technologies and principles that make their products more competitive. Studies show that the customers rather chose quality over price in food product, as shown e.g. in [1] where $72 \%$ of the respondents said that they are more concerned with quality than the price of food. Similar situation is in Serbia, where an agency conducted research that showed that the main reason for deciding on buying a specific product is its quality, with $61 \%$ of interviewed customers [2]. The quality of food products is influenced by 
the source and quality of raw food, but also by the processing and production methods, the packaging and transport of semi-products and finished products. This leads to the necessity for a traceability system that would enable tracking the food product through the whole food chain, and also tracing the food end-product back to its origin, which is recognized as forward and backward traceability.

The EU Regulation 178/2002, which took effect on January $1^{\text {st }} 2005$, set general foundations for traceability requirements in food sector in the EU and at the national level in EU member states [3]. According to this regulation, "traceability means the ability to trace and follow a food, feed, food-producing animal or substance intended to be, or expected to be incorporated into a food or feed, through all stages of production, processing and distribution". This regulation requires traceability of all the abovementioned food products and ingredients, identification of preceding and subsequent participants in the food supply chain, availability of this information to the competent authorities upon demand, and labeling of food products that are ready for the market.

The traceability system is supposed to enable all participants in the food chain to trace food products at all times and to provide full history for the specific product [4], which includes the source of all raw food, treatments to which it was exposed during growing and harvest phase, processing, transport, etc. This system should also protect the consumers from buying out-of-date food, food that contains allergens, religiously prohibited food, etc. In the case of problems with safety and quality of a batch of food products, e.g. appearance of dangerous substances or microorganisms, such a system should enable withdrawal of these products from the market and prevent health disasters.

Traceability systems worldwide have been developed, but they lack standards, companies develop their own systems which are different, and are producing different economic results [5]. Such systems are mainly developed internally within one company or within large food production chains, and they lead to large information gaps between the participants in the food chain. On the other hand, Daives (as cited by [6]) states that $62.2 \%$ of European food companies are small and medium enterprises, they produce the largest amount of food products, but cannot expect the potential benefits from implementing traceability systems in comparison to the cost of work needed for implementing such a system, as they are not connected into a unified information system, which would allow efficient and transparent information flow in supply chains.

One of the challenges in traceability systems are means of storing and transferring data between participants in the food chain. Traditional systems include alphanumerical identification of food products, but such systems require manual identification of products, and are time consuming. Automated identification technologies such as barcode and RFID technologies enable automation of traceability systems and faster and less expensive data collection and transfer. These technologies can thus help overcome problems that exist with traditional traceability systems, and enable full integration of data in a food product chain. RFID and barcode technologies have become widely implemented in many areas due to the increasing availability of devices and systems that enable data collection through 
these technologies. The main challenge in implementing a traceability system based on automated identification technologies is the fact that food products greatly vary from one product to another, regarding the aggregate state, amount of products in a group package, methods of processing, packaging and transportation, storage temperatures, key product data, etc. In order to create a unified system, all of these features have to be taken into account, and the system set up in such a way that it is adaptable to various conditions in food chains.

Taking into account all the abovementioned, several hypotheses can be set: an automated identification system can be developed and applied for food product traceability through different stages; data that are generated in different stages of the food chain can be integrated in one database and thus can be used in subsequent stages; the data generated using automated identification technologies are significant for food safety. Research methodology that is applied for validating the hypotheses includes analysis and synthesis for developing a concept for food traceability based on automated identification technologies, and also experimental validation, that is planned for fulfillment in the next phase of research.

\section{Relationship to Internet of Things}

In the world of globalization and interconnecting various areas into one integrated whole, a very important aspect is the traceability of objects and linking important information into a unique system that allows access to this information to various users, to the appropriate extent. Such systems include systems for food products traceability, which are specific as they can influence human health. A system for food traceability discussed in this paper precisely allows interconnecting various data about food products, from the means, place and time of the farming of raw food, through its processing, packaging, transport and storing, and to its arrival to retailers and end consumers. Such a system should enable storing of all key data in an adequate database, and allow access to this database to various users and according to their position in the food chain and their privileges to enable viewing and/or updating specific food data. This system should be universal and applicable for different types of food products, and to easily provide incorporation of new participants in the process, in order to help the tendency for integration of all relevant data in a unique system. The main purpose of such a system is the availability of all the key data to the end user, which would enable them to more wisely choose products that they are buying, in accordance with their wants, needs, restrictions (e.g. for allergic, vegetarian, diseased consumer). Other very important aim of such a system is the possibility of fast reactions in the case of contaminated batches of food (e.g. with poisonous chemicals or bacteria), where the food can immediately be traced back to its origin and further spread of the contaminated food can be blocked and the already spread food withdrawn. Moreover, such a system can also help producers, as it enables them to recognize the actual demand for products on the market, the amount of their sold products as well as places and time when they are sold, which would let them create better market strategies. 


\section{State of the Art}

As automated identification technologies had become widely used in various areas for tracking different types of objects, systems based on automated identification were also created for traceability of food products.

In [7], a traceability system with the use of standard linear barcodes is presented, on a case company in the baking industry that started applying barcode labels to the cases and pallets in order to improve warehouse inventory system by recording quantity, location and product identification numbers. This system also allowed them to track the subsequent participants in the food chain, and help in risk situations when batches with bad products were accidentally released. The authors state that improved systems for product traceability can be achieved by using two-dimensional barcodes or RFID technology.

In [6], the authors proposed an information infrastructure that enables traceability in the food supply chain by the application of RFID technology. The presented traceability system is cost effective and applicable in the chain of small and medium enterprises, which have limited financial capabilities. Every participant in the food chain has set up RFID readers. The data from food products is collected via RFID readers and sent to the central database through an IP network. The central database can be accessed through a web interface, and each participant in the food supply chain can view the adequate data. The authors state that the greatest potential for RFID application in a food supply chain is in particular in connecting different participants in the chain.

In the meat processing industry [8], traceability systems have to enable tracing meat along the complete chain, back to the origin, as various diseases can influence the quality of meat. The traceability systems have to be considered the highest priority, and not just legislation, in order to ensure safe and high quality meat for the end consumer. In [9] a case traceability system for chicken meat is presented, that integrates RFID technology with the information system. The system is applied through the complete food chain, from the farm, through slaughter house and processing factory, to the retailer. Food traceability data is gathered and registered through RFID readers, and sent to the central database. At specific places there are devices where a consumer can read data from the central database and get the required information.

In Japan [10], an integrated traceability system was developed for identification of agricultural products and storing the key data from production to consumption stages, by the application of RFID technology, mobile phones and web-based network computing. This system enables farmers to input data for methods and materials used in the production stage through their mobile phones, and store them in the production database. Through the distribution stage, data is inputted to the distribution database, and RFID tags or barcodes are applied to the individual products, and they connect each product to the data in the production and the distribution database. Endconsumers can use their internet enabled mobile phones that have integrated RFID or barcode readers to scan data from the RFID tags or barcodes, and access the database with production and distribution data about the particular product. 
The same author [11] has developed a unique database system that integrates the previous production and distribution databases, and a visualization tool for traceability of agricultural products in the food chain. This database system allows changing of the ID tags of products through the chain, and concatenating all the available data about the product. The author states that the major drawback in using the RFID technology in a traceability system is the price of tags and devices used for the transfer of data and the time needed for tagging and reading each product.

In [5] a traceability system is presented for a very expensive Italian cheese, Parmigiano Reggiano. This cheese is produced in large cylindrical pieces. In the presented traceability system, each cheese is labeled with one tag, which precisely identifies the specific cheese. The authors state that this system is applicable and effective as the price of the tag is irrelevant considering the price of the whole cheese. They consider RFID a good solution for traceability of high-value products like cheese or wine, while for the low-priced food products they propose systems still based on alphanumerical codes or barcodes.

\section{$4 \quad$ Research Contribution and Innovation}

The main objective of this paper is to set up a framework for a unique food traceability system that enables transfer of key product data through the entire product transformation chain, which uses automated identification technologies: RFID and two-dimensional barcodes. This framework should be adaptable to various food chains according to the specific demands for traceability of particular products.

RFID technology has many advantages for implementation in automated identification and traceability systems, such as the amount of data that can be contained in a tag, high reading speed of data, possibility of simultaneous reading of multiple tags, possibility of non-contact reading of data, etc. One of the major disadvantages of RFID technology is the price of its implementation and of single tags. This disadvantage affects the use of RFID technology in food product traceability systems, as the price of an RFID tag would greatly affect the price of a single food product. On the other hand, two-dimensional barcodes can store less but still a significant amount of data, and are not costly like RFID tags. They have other disadvantages, including the need for proximity of readers while reading labels, inability of readers to simultaneously read multiple labels, etc. One of the most often used two-dimensional barcodes is QR code, which can store a sufficient amount of data, has very good readability even on small sized labels, and which also has very good readability in case of physical damage of a part of the code. QR codes are mostly used for recording a certain numeric code or a URL to the website that contains information. In this paper the framework for a traceability system is proposed that uses both the RFID and QR codes for food product traceability, in which both the RFID and QR codes contain information about a particular product, which is therefore immediately readable with the use of an adequate reader.

The traceability system framework presented in this paper includes all the possible stages in a food chain, where the potential participants are: primary producers, 
processing industries, transport, retail and the end-consumer (Fig. 1). In a specific traceability system, it is not necessary that all of the above mentioned participants be present, but the system can consist of any possible combination thereof. Each participant in the chain represents a specific stage in the transformation of food.

At each of the transformation stages, specific data are generated that describe the current processes, methods used, place and time, etc. Each product gets a unique ID code, which is the primary key data for the product. All the important data at a particular stage are assigned to the unique ID code of the product, and recorded in the database, to which each participant in the food chain can connect via an IP based computer network. If the new product is a combination of several ingredients, its unique ID code is also connected to the ingredients' unique ID codes in the database, which enables traceability back to each raw product that is integrated in the current food product. This way, the database contains all the existing data about a specific product, which is also connected to the data originating from its ingredients. Also, accessibility levels must be set, to define to what extent subsequent users can reach specific product data in the database.

Moreover, at each stage, the key data is picked, which is significant for the subsequent participant in the chain, and it is incorporated in the label for each product in the form of a QR code. The QR code is chosen for this application as it can store a sufficient amount of data, and at the same time it is small, but can be read even if greatly damaged, can be placed on a sticker, and printed on many available printers.

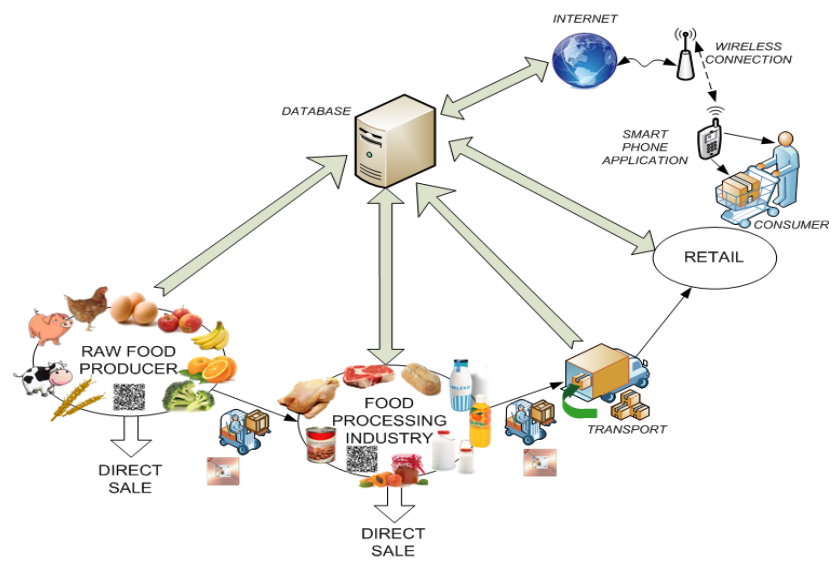

Fig. 1. The concept for food product traceability based on automated identification technologies

An application rarely given to $\mathrm{QR}$ codes is the usage of their possibility of storing a sufficient amount of data in a small space, and not just a numeric code or the address of a website. For example, a QR code sized $25 \times 25 \mathrm{~mm}$ which consists of $69 \times 69$ blocks, can store up to 321 characters of data. The data in the QR code can be accessed by the appropriate reader, such as a dedicated two-dimensional barcode reader or a smart phone with the adequate application. The mandatory data in the food product $\mathrm{QR}$ code is a unique ID code that can connect the user to the correct data in 
the database upon request. The important thing is that here the $\mathrm{QR}$ code is used for tagging each single product, with the corresponding information related solely to it. This enables the subsequent participant in the chain or the end consumer to easily access the most important data about the product without the need for internet connectivity, just by using a smart phone with the application for reading twodimensional barcodes, or a dedicated reader for two-dimensional barcodes. With the increase of everyday usage of smart phones, many users could read such labeling to immediately get the required information about the particular product. If such users install the dedicated application for reading detailed information about products, they can acquire additional information from the database through internet connection, where they can check detailed information about the product or its ingredients at the available accessibility level.

A specific situation occurs in transport, where large amounts of products are simultaneously transferred between the participants in the chain. In the transport stages, products are usually packed in larger group packaging, such as boxes, cases, pallets, barrels, etc. or the products are themselves large. In transport, speed is a very important issue, and if each product or a group packaging should be read with barcode readers, it would require a lot of time. A possible solution to this challenge is to additionally label transport packaging with RFID labels with the required information about the contained products. The RFID technology enables storing of larger amounts of data than two-dimensional barcodes, more RFID tags can be read simultaneously and are readable from a distance with dedicated RFID readers. The disadvantage of RFID technology is its price, which is the reason why it is not yet widely available for identification and traceability systems of individual products. However, this disadvantage is not so significant during the transport stage, as the product packaging is usually large, and contains more products, therefore the use of a single RFID label for the entire packaging would not drastically affect the price of individual products. Furthermore, identification of RFID tags can be done automatically without the need for additional workers for reading labels and registering the products. The implementation of RFID tags during the transport stage would allow immediate registration of sent and received packages, which must be integrated in the whole information system for traceability of particular food products and synchronized between all partners in the chain. This should again be done through the database, where the particular products would get additional data about their new location.

\section{Discussion of Results and Critical View}

This paper provides a framework for a centralized unified system with the application of automated identification technologies for food product traceability. By reviewing the literature, no such complete system could be found, and on the other hand many regulations, both national and worldwide, require complete traceability of food products. There seems to be no single technology that can provide such a complete system for various kinds of existing food chains, which leads to the conclusion that several identification technologies must be integrated into a system where their 
individual advantages will be used, and disadvantages suppressed or even avoided. The proposed framework suggests exactly such a system, where two-dimensional QR codes would be used combined with the RFID technology, using their possibilities for easy integration into various information systems, large memory capacities for the required data, easy printing and application to the products etc. This way the proposed framework can be considered general for use in any food product traceability system.

The suggested traceability system based on automated identification technologies must be implemented in various food product chains, and validation of the proposed framework must be carried out in different conditions in order to reach the real impact that the proposed solution potentially has.

\section{Conclusions and Further Work}

The paper presents a possible conceptual framework for combined application of $\mathrm{QR}$ codes and RFID technology in a traceability system for food products. Such traceability systems are very important as they provide the end consumer with information from all the previous stages of the food product, including the information about the origin of the product ingredients, the means and places of food production and processes, the transportation and storage information, etc. By the implementation of the proposed framework in a traceability system, the end consumers can easily get important information about a particular product, and if interested, they can check further information about the product from the database, which enables them to make better decisions when choosing an appropriate product.

In order to validate the proposed framework, further work must include experimental implementations in as many different food product chains as possible, where also primary producers, food processing industries, transporters and retailers must be included and educated to accept such a system upgrade and integrate it with their current information infrastructures. Only by such real implementations, challenges can be revealed that are not obvious in the theoretical analysis, the existing problems can be solved and the system framework improved.

\section{References}

1. http://www . adweek. com/news/advertising-branding/consumerschoose-quality-over-price-100447 (accessed on October 31, 2012)

2. http://www.gfk.rs/public_relations/press/articles/005465/ind ex.rs.html (accessed on October 31, 2012)

3. Folinas, D., Manikas, I., Manos, B.: Traceability data management for food chains. British Food Journal 108(8), 622-633 (2006)

4. Abad, E., et al.: RFID smart tag for traceability and cold chain monitoring of foods: Demonstration in an intercontinental fresh fish logistic chain. Journal of Food Engineering 93, 394-399 (2009)

5. Regattieri, A., Gamberi, M., Manzini, R.: Traceability of food products: General framework and experimental evidence. Journal of Food Engineering 81, 347-356 (2007) 
6. Kelepouris, T., Pramatari, K., Doukidis, G.: RFID-enabled traceability in the food supply chain. Industrial Management \& Data Systems 107(2), 183-200 (2007)

7. Bar Coding and RFID: The Key to Traceability and Safety in the Foodservice Supply Chain. A ZEBRA Black\&White Paper (2007)

8. Schwagele, F.: Traceability from a European perspective. Meat Science 71, 164-173 (2005)

9. Chen, R.S., Chen, C.C., Yeh, K.C., Chen, Y.C., Kuo, C.W.: Using RFID technology in food produce traceability. WSEAS Transactions on Information Science and Applications 5(11), 1551-1560 (2008)

10. Sugahara, K.: Traceability System for Agricultural Products based on RFID and Mobile Technology. Computer and Computing Technologies in Agriculture II 3, 2293-2301 (2009)

11. Sugahara, K.: A database system and a visualization tool for agricultural product traceability based on RFID technology. In: Proceedings of EFITA/WCCA, pp. 637-643 (2011) 